\title{
Policies for allocation of information in task-oriented groups: elitism and egalitarianism outperform welfarism
}

\author{
Sandro M. Reia, ${ }^{1}$ Paulo F. Gomes, ${ }^{1,2}$ and José F. Fontanari ${ }^{1}$ \\ ${ }^{1}$ Instituto de Fúsica de São Carlos, Universidade de São Paulo, \\ Caixa Postal 369, 13560-970 São Carlos, São Paulo, Brazil \\ ${ }^{2}$ Instituto de Ciências Exatas e Tecnológicas, Universidade Federal de Goiás, 75801-615 Jataí, Goiás, Brazil
}

\begin{abstract}
Communication or influence networks are probably the most controllable of all factors that are known to impact on the problem-solving capability of task-forces. In the case connections are costly, it is necessary to implement a policy to allocate them to the individuals. Here we use an agent-based model to study how distinct allocation policies affect the performance of a group of agents whose task is to find the global maxima of NK fitness landscapes. Agents cooperate by broadcasting messages informing on their fitness and use this information to imitate the fittest agent in their influence neighborhoods. The larger the influence neighborhood of an agent, the more links, and hence information, the agent receives. We find that the elitist policy in which agents with above-average fitness have their influence neighborhoods amplified, whereas agents with below-average fitness have theirs deflated, is optimal for smooth landscapes, provided the group size is not too small. For rugged landscapes, however, the elitist policy can perform very poorly for certain group sizes. In addition, we find that the egalitarian policy, in which the size of the influence neighborhood is the same for all agents, is optimal for both smooth and rugged landscapes in the case of small groups. The welfarist policy, in which the actions of the elitist policy are reversed, is always suboptimal, i.e., depending on the group size it is outperformed by either the elitist or the egalitarian policies.
\end{abstract}

\section{INTRODUCTION}

Solving real-world problems typically entails overcoming obstacles that are beyond the capabilities of a single person, thus requiring the cooperative and coordinated effort of individuals organized in task-oriented groups or epistemic communities, broadly viewed as social systems consisting of producers of knowledge [1, 2]. Information in these systems flows between individuals via social contacts and, in the cooperative problem-solving setting, a key process is imitative learning as expressed in this quote by Bloom "Imitative learning acts like a synapse, allowing information to leap the gap from one creature to another" 3. In the context of epistemic communities, imitative learning is known as exploitation since it corresponds to the search strategy in which the agent borrows known solutions from its network of influencers. This contrasts with exploration, which is seen as the development of novel solutions by the agent [4, 5].

Here we assume that the amount of information an agent can access (i.e., the number of its potential influencers) has a social cost (e.g., it is publicly funded) and we examine the influence of different policies of allocation of resources to the agents on the efficiency of the group to complete the task. These policies are based on the quality of the partial solutions the agents offer to solve the task. To address this problem we use an agent-based model where the agents perform individual trial-and-test searches to probe a fitness landscape (exploration) and imitate a model agent - the best performing agent in their influence neighborhood at the trial (exploitation) 66 8. We consider a scenario where the agents are fixed at the nodes of a random geometric graph [10] and can interact with each other if the distance between them is less than a prespecified threshold. In addition, the agents vary their radiuses of interaction following a prescription or policy that depends only on their (relative) fitness. This feature results in a time-dependent, adaptive directed network that links the agents to their influencers.

The task of the agents is to find the global maxima of smooth and rugged fitness landscapes generated with the NK model [9. The performance of the group is measured by the properly scaled number of trials (or time) required to find those maxima. In particular, the collective search ends when one of the agents finds the global maximum. The amount of information allocated to an agent is determined by the number of agents in its influence neighborhood, which equals the number of incoming connections, and here we consider three distinct policies. The elitist policy in which the agents with above-average fitness amplify their influence neighborhoods whereas the agents with below-average fitness shrink theirs; the welfarist policy in which those actions are reversed; and the egalitarian policy in which the size of the influence neighborhood is the same for all agents, regardless of their fitness. Since the fitness of the agents change as they explore the state space of the fitness landscape, so do their influence neighborhoods, resulting in adaptive directed communication networks, which we characterize through the number and size of their strongly connected components.

The scenario considered here bears a resemblance to the predicament that funding agencies and governments face when allocating resources among alternative competing research programs aiming at solving the same problem [1, 2]. In fact, the priority rule for allocating credit in science by which only the first person to a discovery gets the recognition supports our decision to halt the search the first time an agent finds the global maximum: once a result has been discovered, no value to the 
collective is produced by discovering it again 11, 12. In that sense, welfarism is not a so far-fetched policy for resource allocation since it is arguably a sensible strategy from the perspective of the collective good to foment agents with below-average performances in order to maintain a diversity of approaches to unsolved problems. In addition, similar agent-based models have also been used to study welfare in the context of human cooperation 13 .

We find that for both smooth and rugged landscapes the welfarist policy is always suboptimal, i.e., it is outperformed either by the elitist or by the egalitarian policies. In addition, for small group sizes the egalitarian policy always yields the optimal performance. Except for small groups, the elitist policy is the optimal choice in the case of the smooth landscapes without local maxima, but a too large amplification of the influence neighborhoods of the agents with above-average fitness may seriously harm the performance of groups of intermediate size in the case of rugged landscapes. As expected, high-fitness outliers in the initial randomly generated group are very likely to win the search (i.e., to find the global maximum first) under the elitist policy. Most surprisingly, however, is the finding that even in a situation of strong welfare, the high-fitness outliers of the initial generation are still more likely to become winners, so the welfarist policy cannot reverse the random initial fitness inequality.

The rest of the paper is organized as follows. In Section II] we present a brief description of the NK model of rugged fitness landscapes. In Section III] we describe the rules for setting up the influence neighborhoods of the agents as well as the implementation of the imitative learning search to explore the state spaces of fitness landscapes. In Section ??, we study the performance of this search for both smooth and rugged landscapes focusing on the effects of the group size and of the policies of allocation of information. Finally, Section IV is reserved for our concluding remarks.

\section{NK-FITNESS LANDSCAPES}

The NK model 9] is a computational implementation of fitness landscapes that has been extensively used to study optimization problems in population genetics, developmental biology and protein folding [14. It was introduced originally to model the adaptive evolution process as walks on rugged fitness landscapes and its main advantage, which led to its widespread use in complexity science, is the possibility of tuning the ruggedness of the landscape by changing the two integer parameters that give the model its name, namely, $N$ and $K$. More pointedly, the NK landscape is defined in the space of binary strings of length $N$ and so the parameter $N$ determines the size of the state space, $2^{N}$. The other parameter $K=0, \ldots, N-1$ determines the range of the epistatic interactions among the bits of the binary string and influences strongly the number of local maxima on the land- scape. In time, two bits are said to be epistatic whenever the combined effects of their contributions to the fitness of the binary string are not merely additive 9. In particular, for $K=0$ the corresponding (smooth) landscape has one single maximum whereas for $K=N-1$, the (uncorrelated) landscape has on the average $2^{N} /(N+1)$ maxima with respect to single bit flips [15].

In the NK model, each string $\mathbf{x}=\left(x_{1}, x_{2}, \ldots, x_{N}\right)$ with $x_{i}=0,1$ has a fitness value $\Phi(\mathbf{x})$ that is given by the average of the contributions of each component $i$ in the string, i.e.,

$$
\Phi(\mathbf{x})=\frac{1}{N} \sum_{i=1}^{N} \phi_{i}(\mathbf{x})
$$

where $\phi_{i}$ is the contribution of component $i$ to the fitness of string $\mathbf{x}$. It is assumed that $\phi_{i}$ depends on the state $x_{i}$ as well as on the states of the $K$ right neighbors of $i$, i.e., $\phi_{i}=\phi_{i}\left(x_{i}, x_{i+1}, \ldots, x_{i+K}\right)$ with the arithmetic in the subscripts done modulo $N$. It is assumed, in addition, that the functions $\phi_{i}$ are $N$ distinct real-valued functions on $\{0,1\}^{K+1}$ and, as usual, we assign to each $\phi_{i}$ a uniformly distributed random number in the unit interval [9]. Because of the randomness of $\phi_{i}$, we can guarantee that $\Phi \in(0,1)$ has a unique global maximum and that different strings have different fitness values.

For $K=0$ there are no local maxima and the sole maximum of $\Phi$ is easily located by picking for each component $i$ the state $x_{i}=0$ if $\phi_{i}(0)>\phi_{i}(1)$ or the state $x_{i}=1$, otherwise. However, for $K>0$ finding the global maximum of the NK model is a NP-complete problem 16, which means that the time required to solve the problem using any currently known deterministic algorithm increases exponentially fast with the length $N$ of the strings. The increase of the parameter $K$ from 0 to $N-1$ decreases the correlation between the fitness of neighboring strings (i.e., strings that differ at a single component) in the state space and for $K=N-1$, those fitness values are uncorrelated [17.

Since the functions $\phi_{i}$ in eq. (1) are random, the ruggedness measures (e.g., the number of local maxima) of a particular realization of a NK landscape are not fixed by the choice of the parameters $N$ and $K>0$. In fact, those measures can vary considerably between landscapes with the same values of those parameters [9], which implies that the performances of search heuristics that rely on the local correlations of the fitness landscape will depend on the particular realization of that landscape. Thus, in order to highlight the role of the parameters that are relevant to our goal of exploring the effects of the policies of allocation of information to the agents on group performance, here we compare the performance of the groups for the same realizations of the NK fitness landscapes. In particular, we consider two types of NK landscapes: smooth landscapes with $N=12$ and $K=0$, and rugged landscapes with $N=12$ and $K=4$. For fixed $N$, all NK landscapes with $K=0$ are equivalent and so we can consider a single realization of the smooth 
NK landscapes without lack of generality. For $K=4$, however, we must average the group performance over an ensemble of landscapes in order to obtain statistical meaningful results. To guarantee that the groups solve the same tasks we generate and store a set of 30 landscape realizations with parameters $N=12$ and $K=4$ so the same landscape realizations are used for different parameters of the imitative learning search. The minimum and the maximum number of maxima in the landscape realizations of our ensemble are 31 and 56, respectively, whereas the mean number of maxima is 46.5 .

\section{IMITATIVE LEARNING SEARCH}

We consider a system of $M$ agents placed in a square box of linear size $L$ with periodic boundary conditions. In the initial configuration, the coordinates $x$ and $y$ of each agent are chosen randomly and uniformly over the length $L$. The density of agents $\rho=M / L^{2}$, which we fix to $\rho=1$ throughout the paper, yields the relevant spatial scale to measure the distance between agents on the square box. In fact, since the effective area of an agent is $1 / \rho$, the quantity $d_{0}=1 / \sqrt{\rho}$ can be viewed as the linear size or, for short, the size of an agent and it will be our standard to measure all distances in our study. We note that the fixed value of the density $\rho$ is inconsequential, provided that we use $d_{0}$ as the standard for measuring distances in the square box. Each agent is represented by a binary string of length $N$, whose bits are initially drawn at random with equal probability for 0 and 1 , so that each agent has an associated fitness value $\Phi_{k}$ with $k=1, \ldots, M$. The fitness of the agents change with time as they explore the NK-fitness landscape aiming at finding its global maximum by flipping bits following the rules of the imitative learning search [7] as will be described next. Henceforth we will use the terms agent and string interchangeably.

The influence neighborhood of agent $k$ is comprised of all the agents located inside the circle of radius $d_{k}$ centered at the spatial coordinates of agent $k$. It is among those agents that agent $k$ will select a model to imitate. Here we consider the prescription

$$
d_{k}=d_{0} \exp \left[\alpha\left(\Phi_{k} / \bar{\Phi}-1\right)\right]
$$

where $\bar{\Phi}=\sum_{k=1}^{M} \Phi_{k} / M$ is the mean fitness of the group at time $t$ and, for the sake of clarity, we have omitted the dependence on $t$ of the quantities $d_{k}, \Phi_{k}$ and $\bar{\Phi}$. The parameter $\alpha$ determines the radius of the influence neighborhood of each agent according to its relative fitness. For $\alpha>0$, agents with fitness higher than average have a large influence neighborhood, i.e., they can see and eventually copy more agents in the group, whereas the agents with fitness lower than average have their influence neighborhoods downsized and are likely to become isolated for large $\alpha>0$. We refer to this choice as the elitist policy, since the high-fitness agents

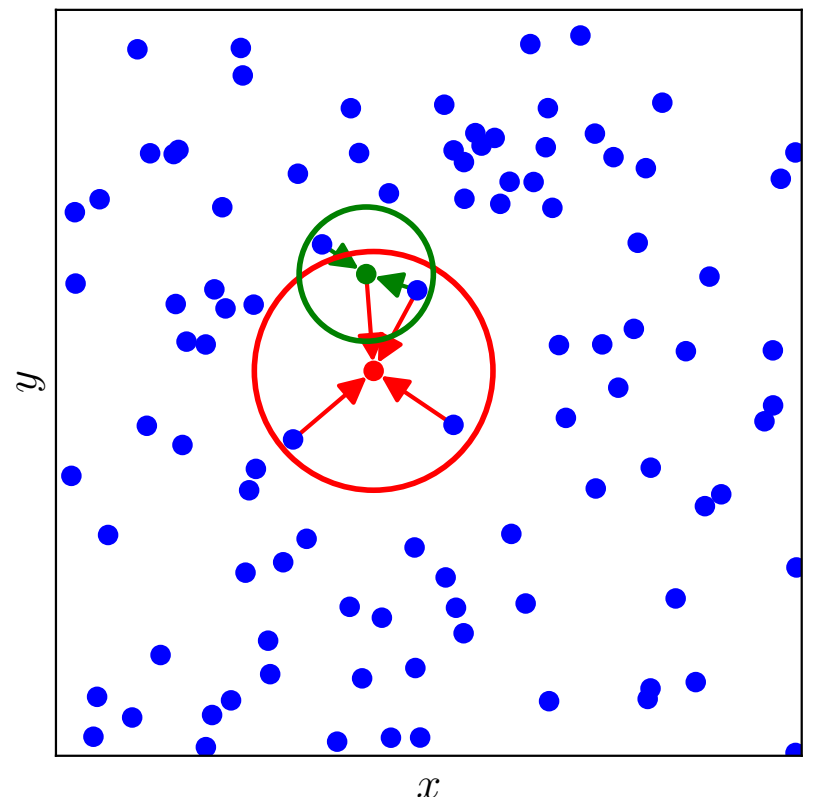

FIG. 1. Snapshot of a system of $M=100$ agents, represented by the bullets, distributed randomly in a square box with density $\rho=1$. The influence neighborhoods are shown for two selected agents only and are highlighted by circles of different colors. This example shows that the resulting influence network is a directed network.

have more opportunities to further improve their fitness through imitation. For $\alpha<0$ the situation is reversed so that the lower-than-average fitness agents have their neighborhoods amplified and the above-than-average fitness agents have theirs curtailed. We refer to this choice as the welfarist policy. The case $\alpha=0$, where the sizes of the influence neighborhoods are the same for all agents, corresponds to an egalitarian policy.

This scenario is illustrated in Fig. 1 that shows a snapshot of a system of $M=100$ agents in the square box. Henceforth we refer to the network created by the union of the influence neighborhoods of all agents as the influence network. This directed network reduces to the classic undirected random geometric graph for $\alpha=0$. The random geometric graph was originally introduced to model wireless communication networks [10] and it was recently used as a face-to-face network in the modeling of the dynamics of human interactions [18] as well as in the study of the effects of mobility on cooperative processes [19].

The dynamics begins with the selection of a target agent at random, say agent $k$, at time $t=0$ and proceeds as follows. A circle of radius $d_{k}$ is drawn around the target agent so that its influence neighborhood is determined (see Fig. 1). If the influence neighborhood is empty, i.e., there is no agent within a distance $d_{k}$ from agent $k$, or all agents in the influence neighborhood have fitness lower than or equal to the fitness of the agent $k$, then this agent simply flips a bit at random. We re- 
call that due to the nature of the NK-fitness landscape the fitness are real-valued random variables - two agents that have the same fitness must be identical (clones). If there are agents with fitness higher than the fitness of the target agent in its influence neighborhood, there are two possibilities of action. The first action, which happens with probability $1-p$, consists of flipping a bit at random of the target string as before. Through the repeated application of this action, the agents can produce all the $2^{N}$ binary strings starting from any arbitrary string, which guarantees that the global maximum will eventually be reached for $p<1$. The second action, which happens with probability $p$, is the imitation of a model string, which is the string of highest fitness in the influence neighborhood of the target agent. In this case, the model and the target strings are compared and the different bits are singled out. Then the target agent selects at random one of the distinct bits and flips it so that this bit is now the same in both strings. Hence, imitation results in the increase of the similarity between the target and the model agents, which may not necessarily lead to an increase of the fitness of the target agent.

The parameter $p \in[0,1]$ is the imitation probability, which we assume is the same for all agents (see [20] for the relaxation of this assumption). The case $p=0$ corresponds to the baseline situation where the $M$ agents explore the state space independently of each other. The case $p=1$ corresponds to the situation where only the model strings explore the state space through random bit flips, whereas the other strings simply follow the models in their influence neighborhoods. The imitation procedure described above was borrowed from the incremental assimilation mechanism used to study the influence of external media [21 23] in Axelrod's model of social influence 24. This is the main feature that distinguishes our model from previous exploration (random bit flips) and exploitation (copy of fittest agent) models in which the copy mechanism is non-incremental so that the target agent is replaced by the model agent [4]. As expected, this non-incremental mechanism may permanently trap the search in the local maxima of the fitness landscape.

After the target agent is updated, which means that exactly one bit of its string is flipped, we increment the time $t$ by the quantity $\Delta t=1 / M$. Then another agent is selected at random and the procedure described above is repeated. We note that during the increment from $t$ to $t+1$, exactly $M$ string operations are performed, though not necessarily by $M$ distinct agents. The search ends when one of the agents finds the global maximum and we denote by $t^{*}$ the halting time. Here we measure the efficiency of the search by the total number of agent updates necessary to find the global maximum (i.e., $M t^{*}$ ), which is essentially the computational cost of the search. Since $t^{*}$ typically scales with the size of the solution space $2^{N}$, it is convenient to present the results in terms of the rescaled computational cost, defined as

$$
C=M t^{*} / 2^{N}
$$

In the case of the independent search $(p=0)$, the ruggedness of the landscape has no effect on the efficiency of the search, which depends only on the length of the strings $N$ and on the group size $M$. In fact, in this case it can be shown that the mean rescaled computational cost is given by

$$
\langle C\rangle=\frac{M}{2^{N}\left[1-\left(\lambda_{N}\right)^{M}\right]},
$$

where $\lambda_{N}$ is the second largest eigenvalue of a tridiagonal stochastic matrix $\mathbf{T}[7]$. The notation $\langle\ldots\rangle$ stands for the average over independent searches on the same landscape. Notice that $\left\langle t_{M}^{*}\right\rangle=1 /\left[1-\left(\lambda_{N}\right)^{M}\right]$ is the expected number of trials for a group of $M$ independent agents to find the global maximum. In particular, for $N=12$ we have $\lambda_{12} \approx 0.99978$ and $\left\langle t_{1}^{*}\right\rangle \approx 4545$. Since $\left(\lambda_{12}\right)^{M} \approx e^{-M\left(1-\lambda_{12}\right)}$ we have $\langle C\rangle \approx\left\langle t_{1}^{*}\right\rangle / 2^{12} \approx 1.11$ for $M \ll\left\langle t_{1}^{*}\right\rangle$ and $\langle C\rangle \approx M / 2^{12}$ for $M \gg\left\langle t_{1}^{*}\right\rangle$.

The assessment of the performance of the imitative learning search is done by comparing its mean computational cost with the cost of the independent search, which is approximated very well by the constant $\langle C\rangle \approx 1.11$ for the typical group sizes $M$ considered in the paper.

As pointed out before, the performance of the imitative search is measured by the mean computational cost $\langle C\rangle$, which is estimated by averaging the computational cost defined by eq. (3) over $10^{4}$ searches on the same landscape realization. For the rugged landscapes, the resulting cost is further averaged over the set of 30 landscape realizations. Since our main concern is the effect of the resource allocation policies on group performance, we will fix the imitation probability to $p=0.5$ and vary the group size $M$ and the parameter $\alpha$ that appears in eq. (2) and determines the strength with which the elitist $(\alpha>0)$ and welfarist $(\alpha<0)$ policies are enforced, i.e., the value of $\alpha$ determines how the radius of the influence neighborhood of an agent is affected by its relative fitness.

\section{A. Smooth Landscape}

The NK fitness landscape with $K=0$ is an additive landscape (i.e., the fitness of a string is given by the sum of the fitness of its components) that exhibits a single maximum. For the particular realization we consider here, the fitness of the maximum is $\Phi^{\max }=0.559$, whereas the average fitness of the landscape is $\Phi^{\text {av }}=0.415$. The mean computational cost of the imitative search for a landscape with $N=12$ and $K=0$ is shown in Figs. 2 and 3 , where the independent variable is $M$ and $\alpha$, respectively. As already pointed out, these results are not dependent on the realization of the smooth landscape.

It is convenient to begin the analysis of Fig. 2 with the results for $\alpha=30$, where we observe an initial decrease of the computational cost with increasing $M$ until 


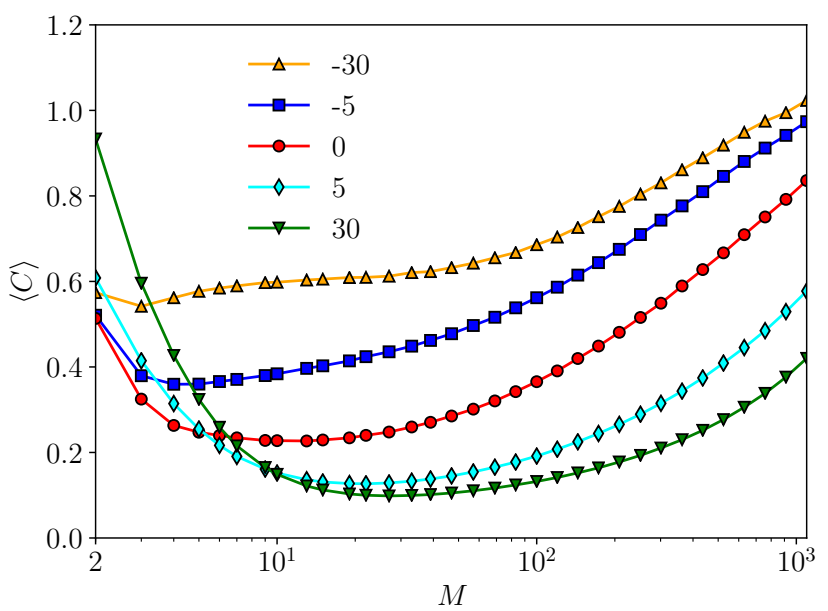

FIG. 2. Mean computational cost $\langle C\rangle$ as function of the group size $M$ for the imitative search on a smooth landscape. The imitation probability is $p=0.5$ and the strength with which the information allocation policies are enforced is $\alpha=-30,-5,0,5,30$ as indicated. The parameters of the NK landscape are $N=12$ and $K=0$.

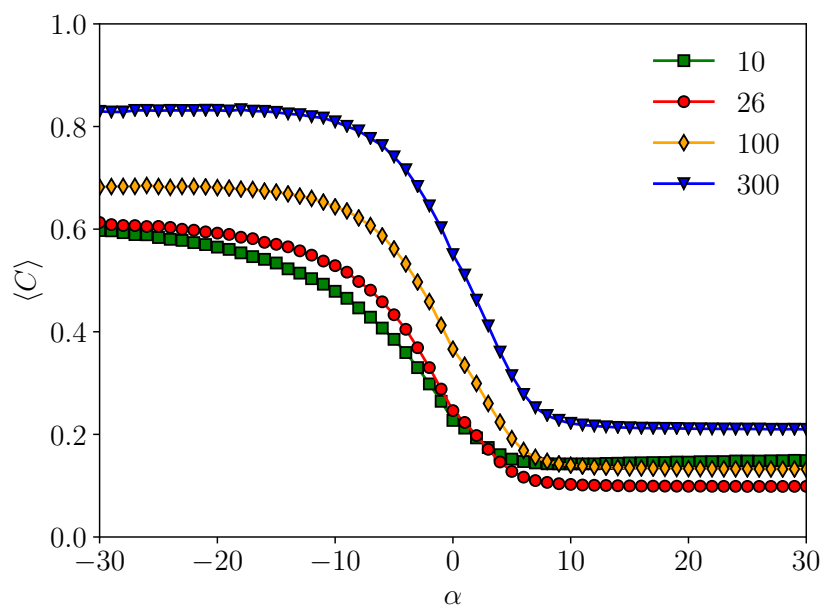

FIG. 3. Mean computational cost $\langle C\rangle$ as function of the strength $\alpha$ with which the information allocation policies are enforced for group sizes $M=10,26,100,300$ as indicated. The imitation probability is $p=0.5$ and the parameters of the NK landscape are $N=12$ and $K=0$.

it reaches a minimum at the optimal group size $M=26$. The subsequent increase of the cost for $M$ greater than this optimum is probably due to the concentration of the strings in the vicinity of the model string and the consequent production of clones that end up reducing the efficiency of the search [7. The optimal group size decreases and the group performance degrades with decreasing $\alpha$. For instance, for $\alpha=-30$, the mean computational cost reaches its minimum value at $M=3$.

Figure 3 shows the computational cost against $\alpha$ and reveals more clearly the interesting result that the group performance improves with increasing $\alpha$, provided that $M$ is not too small. This means that for the imitative search on a smooth landscape it is advantageous to allow the above-average fitness agents to enlarge their influence neighborhoods so they can inspect and eventually imitate more agents in the group. This elitist policy increases the chances of improvement of the agents which already have a high fitness and decreases those of the low-fitness agents, similarly to the so-called Matthew principle in which the rich get richer and the poor get poorer [25]. The opposite, welfarist policy in which the below-average agents enlarge their neighborhoods (i.e., $\alpha<0$ ) results in a much poorer performance, as illustrated in Figs. 2 and 3. Interestingly, however, for small groups, say $M<5$ in Fig. 2, the best performance is achieved for the egalitarian policy $(\alpha=0)$ where the sizes of the influence neighborhoods are not dependent on the fitness of the agents (i.e., $d_{k}=d_{0}$ for $k=1, \ldots, M$ ). To conclude the analysis of the group performance, we stress that the imitative search always outperforms the independent search for the smooth landscape.

It is instructive to look into the characteristics of the agent that found the global maximum and, consequently, halted the search. We refer to this agent as the winner. A useful quantity in this context is the distribution of probability of the number of agents in the winner's influence neighborhood, $\Omega_{w}=0, \ldots, M-1$, at the instant just before it finds the global maximum, which we show in the upper panel of Fig. 4. For the purpose of comparison, we show in the lower panel of Fig. 4 the distribution of probability of the number of agents in the influence neighborhood of a randomly selected agent at the same instant. We note that $P(\Omega)$ gives effectively the distribution of the sizes of the influence neighborhoods at the trial just before the search halts. For $\alpha<0$, these results indicate that the winner is very likely to be an isolated agent (i.e., $\Omega_{w}=0$ ), as expected, though there are little more than $10 \%$ of isolated agents when the search halts. In fact, since the winner must differ of exactly one bit from the global maximum just before it flips the discordant bit, its fitness must be high and, consequently, its influence neighborhood must be small. The same reasoning applies for $\alpha>0$, so the winner is very likely to be connected to all the other agents (i.e., $\Omega_{w}=M-1$ ), although, in this case, there are very few highly connected agents in the group as shown in the lower panel of Fig. 4. Hence, regarding the sizes of their influence neighborhoods and provided an information allocation policy is enforced (i.e., $\alpha \neq 0$ ), the winners are atypical agents just before they find the global maximum.

In order to investigate whether the winners had an edge in the initial random setup of the group, we calculate the difference between the fitness of the winners at time $t=0, \Phi_{w}(0)$, and the initial mean fitness of the group, $\bar{\Phi}(0)$, for each search. Then we average this difference over the $10^{4}$ searches and show the result $\left\langle\Phi_{w}(0)-\bar{\Phi}(0)\right\rangle$ in Fig. 5. Here the notation $\langle\ldots\rangle$ represents an average over different searches and, in the 

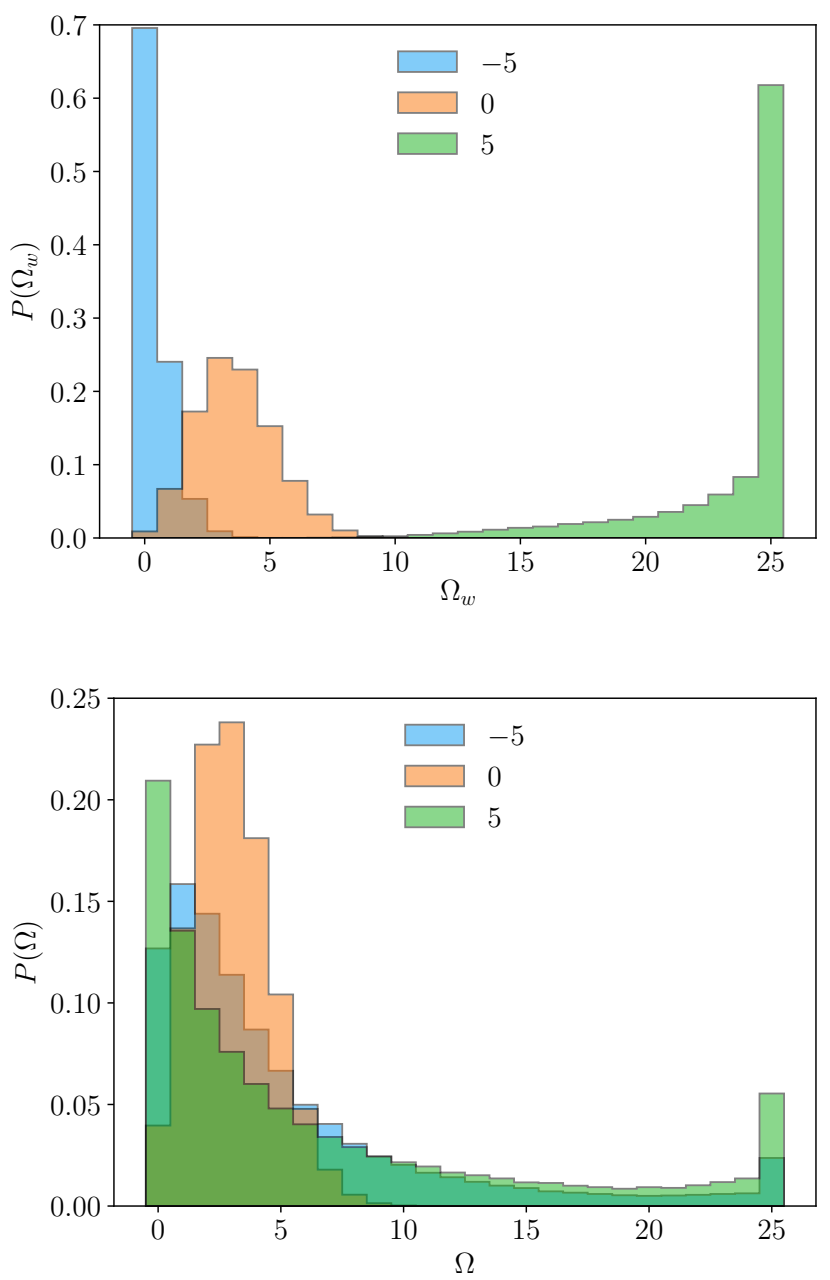

FIG. 4. Probability distribution of the number of agents in the influence neighborhood of the winner (upper panel) and of a randomly selected agent (lower panel) at the instant just before the winner finds the global maximum for $M=26$ and $\alpha=-5,0,5$ as indicated. The imitation probability is $p=0.5$ and the parameters of the NK landscape are $N=12$ and $K=0$.

case of rugged landscapes, over landscape realizations, whereas ... stands for an average over the agents in the group. The effect of the group size $M$ can be understood by noting that for small $M$ the chances that an agent an outlier- is assigned a high fitness value at $t=0$ are very meager and so, in this case, $\Phi_{w}(0)$ does not differ much from the group average $\bar{\Phi}(0)$. As $M$ increases, the chances that an outlier appears increase and the result that $\left\langle\Phi_{w}(0)-\bar{\Phi}(0)\right\rangle>0$ indicates that those outliers are more likely to be the winners, regardless of the value of $\alpha$. The dependence of $\left\langle\Phi_{w}(0)-\bar{\Phi}(0)\right\rangle$ on $\alpha$ for fixed $M$ is more instructive, as it shows that the elitist policy $(\alpha>0)$ practically selects the future winners already in the initial generation by allowing the fittest agent to reap all the benefits of imitative learning. This is again an illustration of the Matthew principle in action.

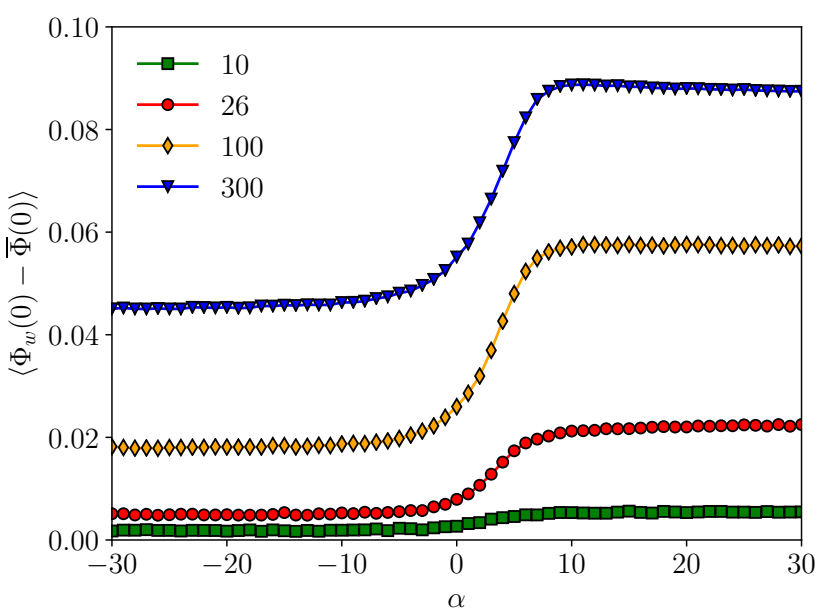

FIG. 5. Average difference between the initial fitness of winner and the initial mean fitness of the group $\left\langle\Phi_{w}(0)-\bar{\Phi}(0)\right\rangle$ as function of the strength $\alpha$ with which the information allocation policies are enforced for group sizes $M=$ $10,26,100,300$ as indicated. The imitation probability is $p=0.5$ and the parameters of the NK landscape are $N=12$ and $K=0$.

Since for $\alpha \neq 0$ the sizes of the influence neighborhoods vary as the agents explore the fitness landscape through the imitative search, it is of interest to characterize the influence networks when the search halts. As pointed out, an influence network, which is formed by the union of the influence neighborhoods of all agents, is a directed graph where the agents are the nodes and neighboring agents are connected by directed edges (see Fig. 1). Since the nub of the imitative search is to spread useful information (i.e., bits that increase fitness) among the members of the group, we focus only on the connectivity properties of the influence networks.

We recall that a directed graph is said to be strongly connected if every node is reachable from every other node, and a strongly connected component (SCC) of a directed graph is a maximal strongly connected subgraph 26 . Thus, following the usual line of analysis used to study percolation [27, we consider the number $\mathcal{N}_{c}$ of SCCs and the size $\mathcal{G}_{c}$ of the largest SCC of the influence network when the search halts. By the size of a SCC we mean the number of nodes that belong to it. Figure 6 shows these two quantities in a properly normalized form, viz., $n_{c}=\mathcal{N}_{c} / M$ and $g_{c}=\mathcal{G}_{c} / M$. The fraction of SCCs reaches its minimum value for $\alpha=0$ and is slightly asymmetric around that point, i.e., there is a bit more SCCs for positive than for negative $\alpha$. The size of the largest SCC exhibits this slight asymmetry too. The quasi-invariance of the properties of the SCCs to change in the sign of $\alpha$ is expected since when $\alpha$ is replaced by $-\alpha$ there is essentially an interchange of the influence neighborhoods between agents whose fitness are above and below the mean fitness of the group by similar amounts. 

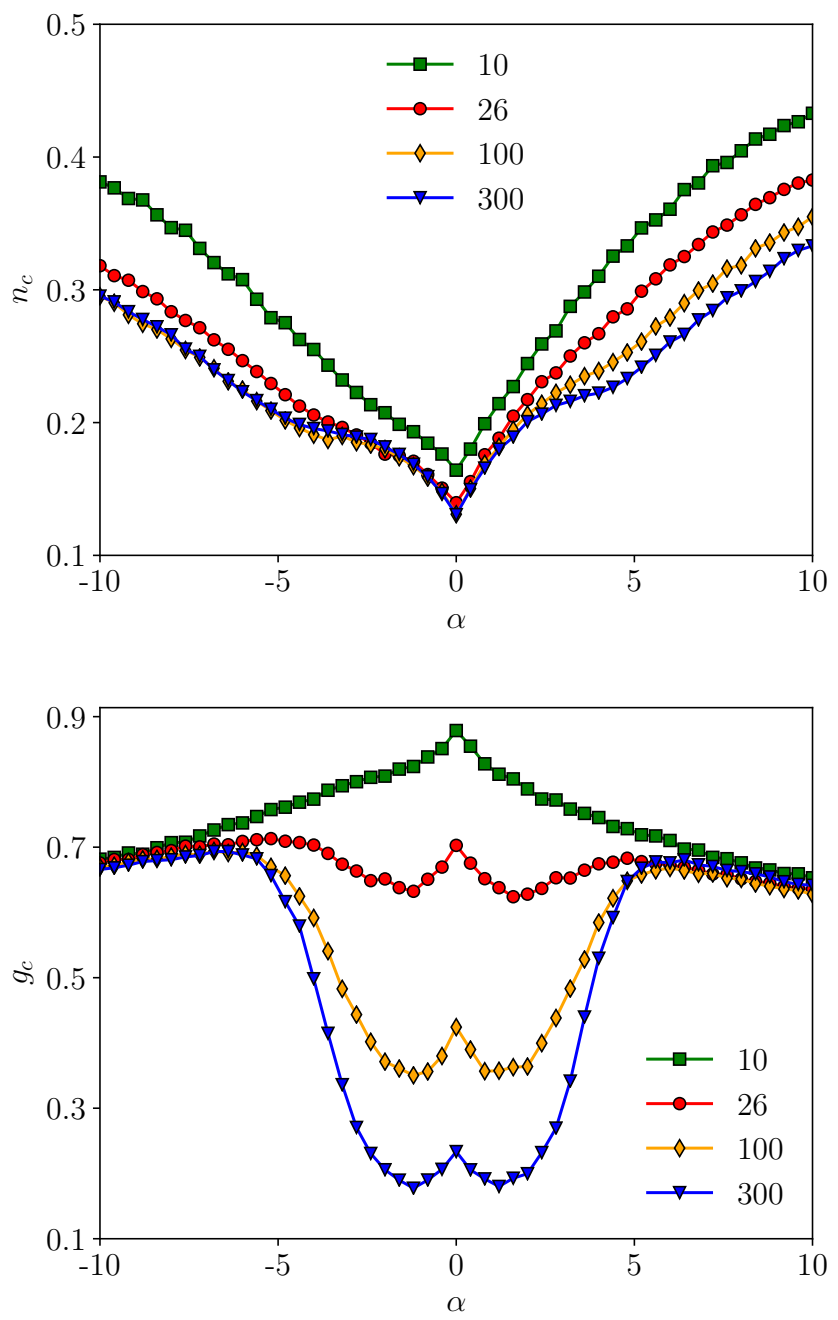

FIG. 6. Fraction of strongly connected components $n_{c}$ (upper panel) and fraction of agents in the largest strongly connected component $g_{c}$ (lower panel) for group sizes $M=$ $10,26,100,300$ as indicated. The imitation probability is $p=0.5$ and the parameters of the NK landscape are $N=12$ and $K=0$.

The fact that the largest SCC occurs for $\alpha=0$ for small group sizes and contains about $90 \%$ of the agents may be the reason that the egalitarian policy is optimal in this situation (see Fig. 2). For large $M$, however, there is no obvious link between $g_{c}$ and the computational cost, since $g_{c}$ is practically the same for $\alpha=10$ and $\alpha=-10$, but the costs are very distinct (see Fig. 3).

It is interesting to note that, for $|\alpha|<5, g_{c}$ goes to zero as $M$ increases so that the directed graph is composed of a macroscopic number of microscopic SCCs since $n_{c}$ is nonzero. For $|\alpha|>5$ we also have a macroscopic number of SCCs, but now at least one component is macroscopic. In time, a quantity is macroscopic (microscopic) if it grows linearly (sublinearly) with $M$ in the asymptotic limit $M \rightarrow \infty$. Moreover, we observe in Fig. 6 that there is a region where both $g_{c}$ and $n_{c}$ increase with in-

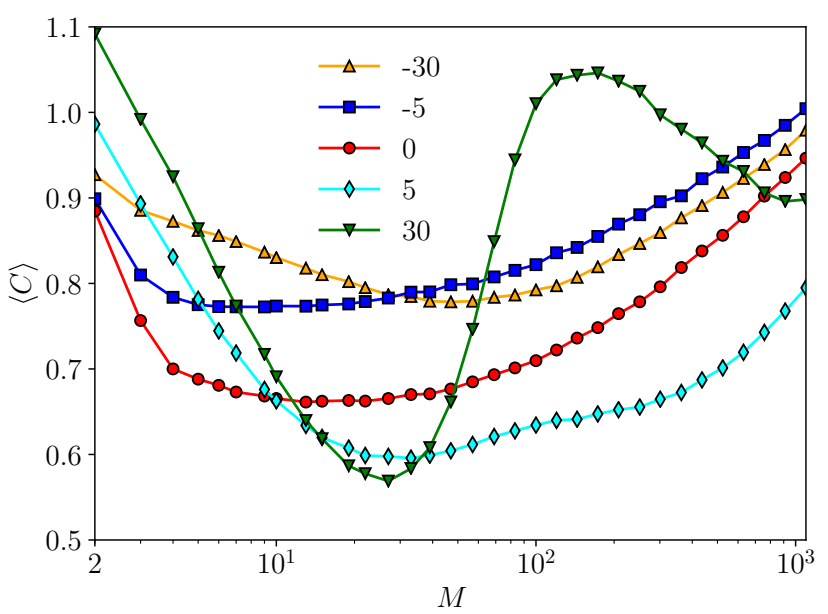

FIG. 7. Mean computational cost $\langle C\rangle$ as function of the group size $M$ for the imitative search on rugged landscapes. The imitation probability is $p=0.5$ and the strength with which the information allocation policies are enforced is $\alpha=$ $-30,-5,0,5,30$ as indicated. The parameters of the NK landscapes are $N=12$ and $K=4$.

creasing $|\alpha|$. This means that while the largest SCC takes in new nodes, other components break into small components. This is expected considering the dual effect of increasing $|\alpha|$ which increases the influence neighborhood of some agents and decrease of others, probably producing isolated agents which would explain the increase of $n_{c}$.

\section{B. Rugged Landscapes}

Since even the less rugged landscape in our ensemble of 30 NK-fitness landscapes with parameters $N=12$ and $K=4$ has 31 maxima, finding the unique global maximum of each realization in this set poses a difficult challenge to any hill-climbing type of search strategy. In fact, the presence of those local maxima makes the computational cost of the imitative search very susceptible to the choice of the group size and of the policy to allot information to the agents. Figures 7 and 8 illustrate the intricacies of this choice.

Whereas the best performance shown in Fig. 7 is achieved by the highly elitist policy $(\alpha=30)$ for $M \approx 26$, this policy gives the worst performance for groups of small and intermediate sizes. In particular, the peak of the cost observed for groups of intermediate size can be viewed as a groupthink-like phenomenon [28] that happens because the agents are trapped in high fitness local maxima far away from the global maximum. The cost to escape those maxima can be very high due to the attractive effect of the clones of the model string [7. Nevertheless, the computational cost of the imitative search is always lower than the cost of the independent search, which is $\langle C\rangle \approx 1.1$ for the range of $M$ shown in the figure. This 


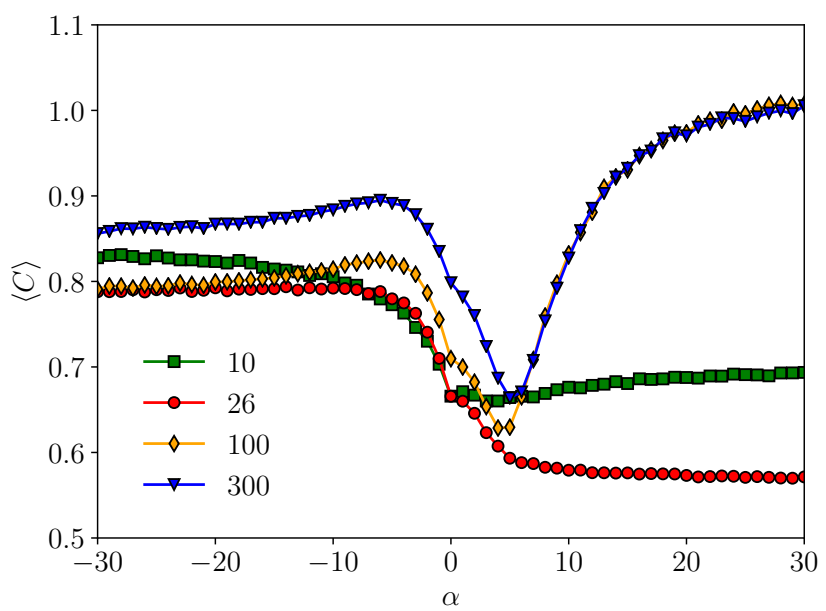

FIG. 8. Mean computational cost $\langle C\rangle$ as function of the strength $\alpha$ with which the information allocation policies are enforced for group sizes $M=10,26,100,300$ as indicated. The imitation probability is $p=0.5$ and the parameters of the NK landscapes are $N=12$ and $K=4$.

contrasts with the results for the fully connected system for which the maximum cost of the imitative search is much higher than the cost of the independent search [7. The reason is that the prescription (2) for the radiuses of the influence neighborhoods of the agents introduces an effective diversity in the imitation probabilities of the agents. In fact, since isolated agents can only flip bits at random their effective probability of imitation is zero. It is the presence of those agents in the group that prevents the trapping of the entire group in a local maximum, which would then characterize a full-blown groupthink event 20.

Interestingly, for small group sizes, the best performance is achieved by the egalitarian policy $(\alpha=0)$ as in the case of the smooth landscape. In fact, for small group sizes the performances of the distinct allocation policies is little influenced by the ruggedness of the landscapes (see Figs. 2 and 7).

Figure 8 shows that the welfarist policy $(\alpha<0)$ is always suboptimal, regardless of the value of $M$. By suboptimal we mean that either $\alpha=0$ or $\alpha>0$ yield a better performance for a fixed $M$. This conclusion holds true for smooth and rugged landscapes as well (see Figs. 3 and 8 .

Figure 9 shows the distribution of the number of agents in the influence neighborhood of the winner and of a randomly selected agent at the instant just before the winner finds the global maximum. The winner is almost certainly isolated for $\alpha<0$ or fully connected for $\alpha>0$, although the size of the influence neighborhood of a randomly selected agent is very little affected by the sign of $\alpha$. Use of the elitist policy $(\alpha>0)$ gives an advantage to high-fitness outliers produced in the initial setup of the group as shown in Fig. 10, but it is not as significant as in the case of the smooth landscape (see Fig. 5 ).
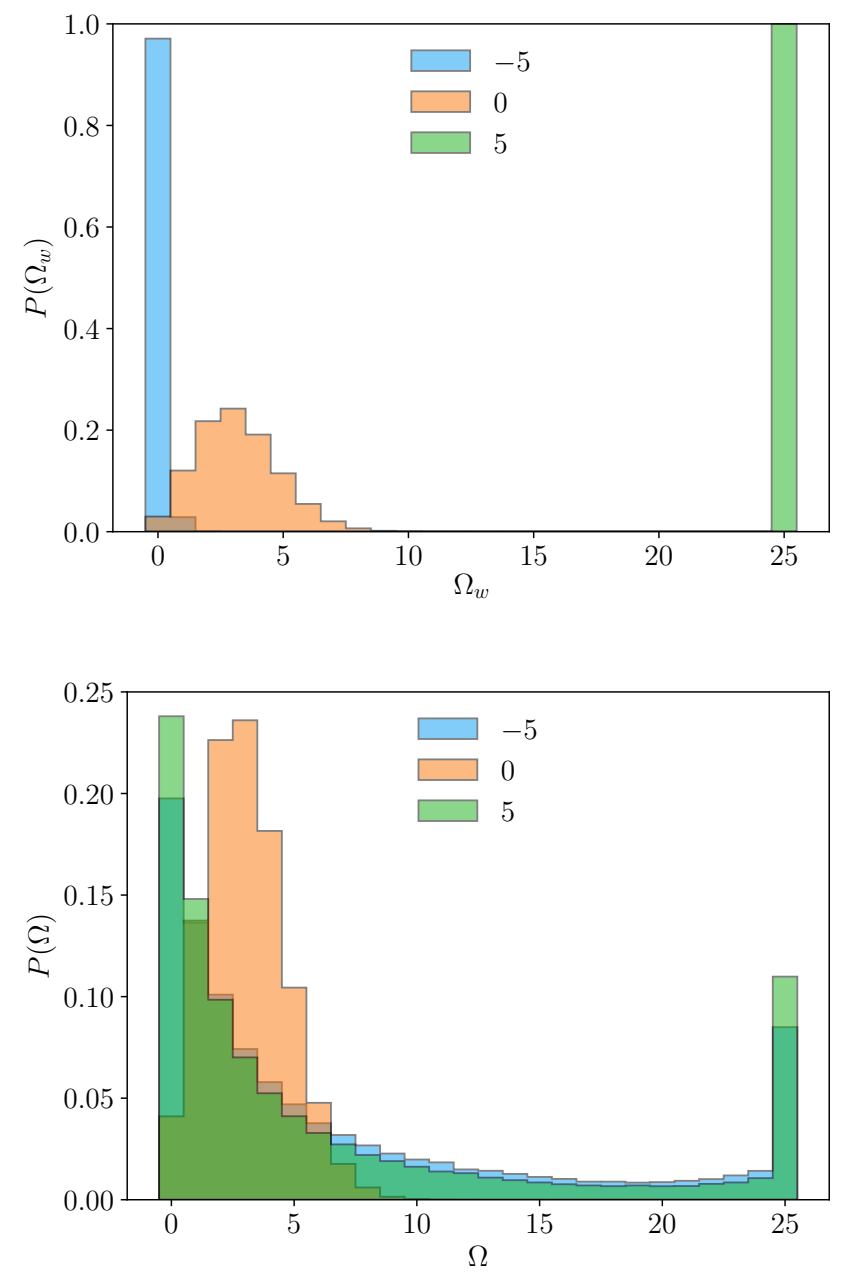

FIG. 9. Probability distribution of the number of agents in the influence neighborhood of the winner (upper panel) and of a randomly selected agent (lower panel) at the instant just before the winner finds the global maximum for $M=26$ and $\alpha=-5,0,5$ as indicated. The imitation probability is $p=0.5$ and the parameters of the NK landscapes are $N=12$ and $K=4$.

This is probably because enlarging the influence neighborhood of an agent is not necessarily advantageous for the group performance as the fitness of the model agents are not strongly correlated to the distances to the global maximum as happens for the smooth landscape.

To conclude, we note that the quantities $n_{c}$ and $g_{c}$ for the rugged landscapes are very similar to those shown in Fig. 6 for the smooth landscape. Hence the difficulty of the problem posed to the group does not seem to influence the connectivity properties of the influence networks when the search halts. 


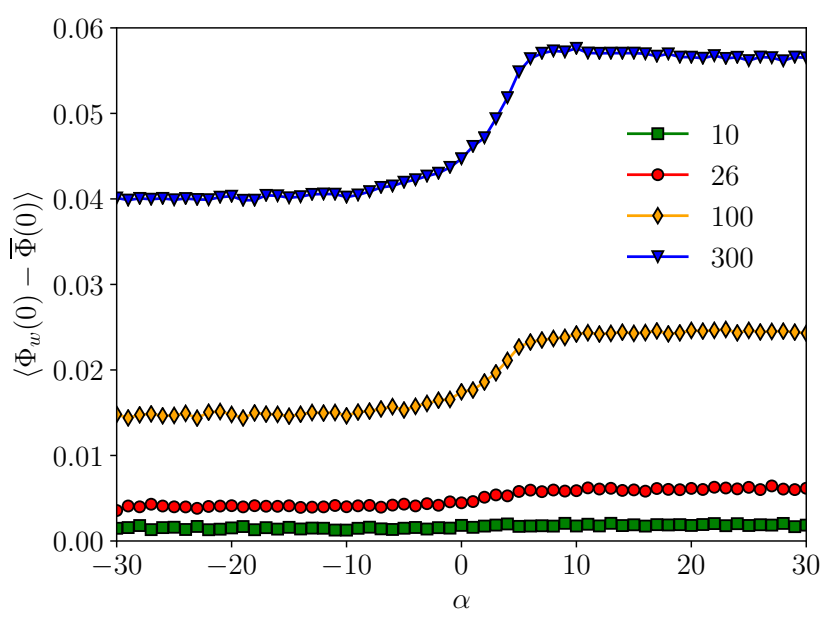

FIG. 10. Average difference between the initial fitness of winner and the initial mean fitness of the group $\left\langle\Phi_{w}(0)-\bar{\Phi}(0)\right\rangle$ as function of the strength $\alpha$ with which the information allocation policies are enforced for group sizes $M=$ $10,26,100,300$ as indicated. The imitation probability is $p=0.5$ and the parameters of the NK landscapes are $N=12$ and $K=4$.

\section{DISCUSSION}

Problem solving by small or large groups of people is a critical issue in modern life as evinced by today's highly successful web-enabled collective intelligence enterprises such as Google and Wikipedia 29. Hence the relevance of understanding the factors that influence the capability of task-oriented groups to solve problems. Here we approach this issue by combining ideas from organizational design 4 and the theory of distributed cooperative problem-solving systems 30].

It has long been realized that the patterns of communication that determine who can communicate with whom in a task-oriented group have a great impact on its problem-solving performance both in the case where cooperation is mandatory to solve the task (e.g., finding the common card in decks distributed to subjects as in Leavitt-Bavelas' experiment 3133) and in the case where a single individual could in principle solve the task (e.g., finding the global maximum of a fitness landscape [34, 35]). However, these studies have focused on imposed or fixed communication patterns, thus excluding a priori the interesting possibility of self-organization of the communication networks.

Here we explore a scenario of flexible communication patterns where immobile agents vary their radiuses of interaction according to the (relative) quality of the solutions they offer to the problem posed to the group, which is to find the global maximum of a NK-fitness landscape. The group performance is measured by a computational cost that essentially tallies the total number of bit flips performed on the $M$ binary strings (i.e., agents) that compose the group until the global maximum is found.
The variation of the sizes of the influence neighborhoods of the agents results in a time-dependent, adaptive directed network that links the agents to their influencers. Since the size of the influence neighborhood of an agent is a measure of the amount of information it can use to decide which bit to flip, it is necessary to establish a policy for allocation of information to the agents based on their fitness. Here we consider three information allocation strategies that are determined by the sign of the parameter $\alpha$ in eq. (2). The first is the elitist policy in which agents with above-average fitness have their influence neighborhoods amplified, whereas agents with below-average fitness have theirs deflated. The second is the welfarist policy in which the actions of the elitist policy are reversed, and the third is the egalitarian policy in which the size of the influence neighborhood is the same for all agents.

Policies for allocation of information are of great importance when the links or connections between individuals are costly, as in the case of social networks of gregarious animals where there is a direct selection pressure to reduce the number of connections between entities because of their building and maintenance costs 36.38. In addition, the view of science as a massive, real-world collective search problem and of scientists as single solutionsearching units 2, 5] brings forth the issue of how to allot resources to competing scientists. Resources that are typically used for 'networking' as in the scenario described here. Moreover, the notions of exploration (discovering new results) and exploitation (borrowing results from others) are not strange to the scientific enterprise. In this context, a natural criterion to allot funds to scientists is their reputations. In the context of searching for the global optimum of a NK-fitness landscape, the relative fitness plays the role of the scientist's reputation, hence our proposal of the prescription (2) to define the radiuses of the influence neighborhoods of the agents.

Somewhat surprising, we find that for small groups the egalitarian policy is optimal for both smooth and rugged landscapes (Figs. 2 and 7). In addition, we find that the elitist policy is optimal for smooth landscapes, provided the group size is not too small. However, this policy produces disastrous results for groups of intermediate sizes in the case of rugged landscapes, which is akin to the groupthink phenomenon of social psychology 28] that results from the lack of opinion diversity among the group members. The welfarist policy, on the other hand, is always suboptimal and, in particular, it is always outperformed by the egalitarian policy.

An interesting and realistic addition to our problem solving-solving scenario is to consider that the members within the group are subject to a social network besides the professional network studied here. The fixedtopology social network could be considered as a second layer of a two-layer network [39], with the first layer being our adaptive influence network. On the one hand, this scenario will prevent the appearance of isolated agents, which could improve the group performance. On the 
other hand, the effective increase of the network connectivity due to the extra layer may degrade the group performance by magnifying the groupthink phenomenon 40. This tradeoff makes the study of the two-layer network scenario an attractive research program.

An appealing finding about the elitist policy regards its potential to select high-fitness outliers in the initial randomly generated group as the winner of the search, i.e., the agent that finds the global maximum first. For both smooth and rugged landscapes, the elitist policy picks winners with a much higher initial mean fitness than those of the other two policies (Figs. 5 and 10, in accordance with the Matthew principle that 'the rich get richer and the poor get poorer'. More interesting, however, is the finding that even in a situation of strong welfare, say $\alpha=-30$ in those figures, where the high-fitness agents are isolated and the low-fitness agents are allowed access to the entire group, the high-fitness outliers of the initial generation are still more likely to become winners, so our welfarist policy cannot reverse the random initial fitness inequality. These conclusions are valid for large groups only, for which there is a reasonable chance of producing a random string with fitness much higher than the average of the group.
The characterization of the influence networks using the distribution of the sizes of the influence neighborhoods (lower panels of Figs. 4 and 9 and the statistics of the strongly connected components (Fig. 6) reveal the rich topology produced by the interplay between the network structure and the imitative search dynamics. We conclude that, except for small groups, some degree of flexibility on the communication patterns among agents can be beneficial to the group performance, provided an elitist policy is enforced with moderate strength.

\section{ACKNOWLEDGMENTS}

The research of JFF was supported in part by Grant No. 2017/23288-0, Fundação de Amparo à Pesquisa do Estado de São Paulo (FAPESP) and by Grant No. 305058/2017-7, Conselho Nacional de Desenvolvimento Científico e Tecnológico (CNPq). SMR was supported by grant 15/17277-0, Fundação de Amparo à Pesquisa do Estado de São Paulo (FAPESP).
[1] S. Reijula, J. Kuorikoski, Modeling epistemic communities, in The Routledge Handbook of Social Epistemology, edited by M. Fricker, P.J. Graham, D. Henderson, N.J.L.L. Pedersen (Routledge, Abingdon, UK, 2019)

[2] P. Kitcher, The Advancement of Science: Science Without Legend, Objectivity Without Illusions (Oxford University Press, New York, 1993)

[3] H. Bloom, Global Brain: The Evolution of Mass Mind from the Big Bang to the 21st Century (Wiley, New York, 2001)

[4] D. Lazer, A. Friedman, Admin. Sci. Quart. 52, 667 (2007)

[5] R.L. Goldstone, M.E. Roberts, W. Mason W, T. Gureckis, Collective Search in Concrete and Abstract Spaces, in Decision Modeling and Behavior in Complex and Uncertain Environments, edited by T. Kugler, J.C. Smith, T. Connolly, Y.-J. Son (Springer, New York, 2008), pp. 277-308

[6] J.F. Fontanari, PLoS ONE 9, e110517 (2014)

[7] J.F. Fontanari, Eur. Phys. J. B 88, 251 (2015)

[8] S.M. Reia, A.C. Amado, J.F. Fontanari, Phys Life Rev. https://doi.org/10.1016/j.plrev.2018.10.004 (2019)

[9] S.A. Kauffman, S. Levin, J. Theor. Biol. 128, 11-45 (1987)

[10] E. Gilbert, J. Soc. Indust. Appl. Math. 9, 533-543 (1961)

[11] R. K. Merton, The sociology of science: Theoretical and empirical investigations (University of Chicago Press, Chicago, 1973)

[12] M. Strevens, J. Philos. 100, 55-79 (2003)

[13] M. Perc, J.J. Jordan, D.G. Rand, Z. Wang, S. Boccaletti, S. Attila, Phys. Rep. 687, 1-51 (2017)

[14] S. A. Kauffman, At Home in the Universe: The Search for Laws of Self-Organization and Complexity (Oxford
University Press, New York, 1995)

[15] H. Kaul, S.H. Jacobson, Math. Program. 108, 475-494 (2006)

[16] D. Solow, A. Burnetas, M. Tsai, N.S. Greenspan, Complex Systems 12, 423-456 (2000)

[17] W. Hordijk, S. A. Kauffman, P. F. Stadler, Theory Biosci. https://doi.org/10.1007/s12064-019-00296-0 (2019)

[18] M. Starnini, A. Baronchelli, R. Pastor-Satorras, Phys. Rev. Lett. 110, 168701 (2013)

[19] P.F Gomes, S.M. Reia, F.A. Rodrigues, J.F. Fontanari, Phys Rev E 99, 032301 (2019)

[20] J.F. Fontanari, EPL 113, 28009 (2016)

[21] Y. Shibanai, S. Yasuno, I. Ishiguro, J. Conflict Res. 45, 80-96 (2001)

[22] J.C. González-Avella, M.G. Cosenza, M. Eguíluz, M. San Miguel, New J. Phys. 12, 013010 (2010)

[23] L.R. Peres, J.F. Fontanari, Europhys. Lett. 96, 38004 (2011)

[24] R. Axelrod, J. Conflict Res. 41, 203-226 (1997)

[25] R.K. Merton, Science 159, 56-63 (1968)

[26] M. Newman, Networks: An Introduction (Oxford University Press, New York, 2010)

[27] D. Stauffer, A. Aharony, Introduction to PercolationTheory (Taylor \& Francis, London, 1992)

[28] I.L. Janis, Groupthink: psychological studies of policy decisions and fiascoes (Houghton Mifflin, Boston, 1982)

[29] T. Malone, R. Laubacher, C. Dellarocas, MIT Sloan Manag. Rev. 51, 1-6, (2010)

[30] B.A. Huberman, Physica D 42, 38-47 (1990)

[31] A. Bavelas, J. Acoustical Soc. Amer. 22, 725 (1950)

[32] H.J. Leavitt, J. Abnorm. Soc. Psych. 46, 38 (1951) 
[33] S.M. Reia, P.F Gomes, J.F. Fontanari, Eur. Phys. J. B 92, 109 (2019)

[34] W. Mason, D.J. Watts, Proc. Natl. Acad. Sci. 109, 764 (2012)

[35] S.M. Reia, S. Herrmann, J.F. Fontanari, Phys. Rev. E 95, 022305 (2017)

[36] J.S. Waters, J.H. Fewell, PLoS ONE 7, e40337 (2012)

[37] C. Pasquaretta, M. Levé, N. Claidière, E. van de Waal, A. Whiten, A.J.J. MacIntosh, M. Pelé, M.L. Bergstrom, C. Borgeaud, S.F. Brosnan, M.C. Crofoot, L.M. Fedigan,
C. Fichtel, L.M. Hopper, M.C. Mareno, O. Petit, A.V. Schnoell, E.P. di Sorrentino, B. Thierry, B. Tiddi, C. Sueur, Sci. Rep. 4, 7600 (2014)

[38] R.H.J.M. Kurvers, J. Krause, D.P. Croft, A.D.M. Wilson, M. Wolf, Trends Ecol. Evol. 29, 326-335 (2014)

[39] M.E. Dickison, M. Magnani, L. Rossi, Multilayer Social Networks (Cambridge University Press, Cambridge, UK, 2016)

[40] J.F. Fontanari, F.A. Rodrigues, Theory Biosci. 135, 101$110(2016)$ 